\title{
Erratum to: Dietary ascorbic acid influences the intestinal morphology and hematology of hybrid sorubim catfish (Pseudoplatystoma reticulatum $\times$ P. corruscans)
}

\author{
Robson Andrade Rodrigues ${ }^{1}$ - Cleujosí da Silva Nunes ${ }^{2}$ • \\ Leticia Emiliani Fantini ${ }^{1}$. \\ Rodrigo Yutaka Dichoff Kasai ${ }^{3}$. \\ Carlos Antonio Lopes Oliveira ${ }^{4}$ - Hamilton Hisano ${ }^{5}$. \\ Cristiane Meldau de Campos ${ }^{1}$
}

\section{Erratum to: Aquacult Int https://doi.org/10.1007/s10499-017-0188-0}

The original version of this article unfortunately contained an error in the authorgroup and affiliation sections.

The supercript of the co-author Cristiane Meldau de Campos should have been 1 not 2. The corrected author group is shown above.

The first and second affiliations should have been "Programa de Pós-Graduação em Zootecnia, Produção Animal no Cerrado-Pantanal, Universidade Estadual de Mato Grosso do Sul (UEMS),

The online version of the original article can be found at https://doi.org/10.1007/s10499-017-0188-0

Robson Andrade Rodrigues robandrod88@gmail.com

1 Programa de Pós-Graduação em Zootecnia, Produção Animal no Cerrado-Pantanal, Universidade Estadual de Mato Grosso do Sul (UEMS), Rodovia Aquidauana/UEMS - Km 12, Aquidauana, MS 79200-000, Brazil

2 Bolsista de Desenvolvimento Científico Regional - DCR (FUNDECT/CNPq), Universidade Estadual de Mato Grosso do Sul (UEMS), Rodovia Aquidauana/UEMS - Km 12, Aquidauana, MS 79200-000, Brazil

3 Pirai Pisicultura, Terenos, MS, Brazil

4 Universidade Estadual de Maringá (UEM), Centro de Ciências Agrárias, Avenida Colombo 5790, Cidade Universitária, Maringá, PR 87020-900, Brazil

5 Embrapa Meio Ambiente, Rodovia SP 340 - Km 127.5, Caixa Postal 69, Jaguariúna, SP 13820-000, Brazil 
Rodovia Aquidauana/UEMS - Km 12 Aquidauana, MS, 79200-000, Brazil" and "Bolsista de Desenvolvimento Científico Regional - DCR (FUNDECT/CNPq), Universidade Estadual de Mato Grosso do Sul (UEMS), Rodovia Aquidauana/UEMS - Km 12 Aquidauana, MS, 79200000, Brazil" not "Programa de Pós-Graduação em Ciência Animal, Faculdade de Medicina Veterinária e Zootecnia - FAMEZ, Universidade Federal de Mato Grosso do Sul - UFMS, Av. Senador Felinto Muller, 2443, Campo Grande, MS 79070-900, Brazil" and "Universidade Estadual de Mato Mato Grosso do Sul (UEMS), Rodovia Aquidauana/UEMS - Km 12, Aquidauana, MS 79200-000, Brazil"

The corrected affiliations are shown in the previous page. 